\title{
Reflux characteristics in patients with gastroesophageal reflux- related chronic cough complicated by laryngopharyngeal reflux
}

\author{
Yiming Yu ${ }^{1,2 \#}$, Siwan Wen ${ }^{1 \#}$, Shengyuan Wang ${ }^{1}$, Cuiqin Shi ${ }^{1}$, Hongmei Ding ${ }^{1}, Z_{\text {Zhongmin Qiu }}{ }^{1}$, Xianghuai \\ $\mathrm{Xu}^{1}, \mathrm{Li} \mathrm{Yu}{ }^{1}$ \\ ${ }^{1}$ Department of Pulmonary and Critical Care Medicine, Tongji Hospital, Tongji University School of Medicine, Shanghai 200065, China; \\ ${ }^{2}$ Department of Pulmonary and Critical Care Medicine, Tongren Hospital, Shanghai Jiaotong University School of Medicine, Shanghai 200336, \\ China \\ Contributions: (I) Conception and design: Y Yu, S Wen, X Xu, L Yu; (II) Administrative support: L Yu; (III) Provision of study materials or patients: \\ Y Yu, S Wen, S Wang, C Shi, H Ding, Z Qiu; (IV) Collection and assembly of data: Y Yu, S Wen, S Wang, C Shi, H Ding, Z Qiu; (V) Data analysis \\ and interpretation: Y Yu, S Wen; (VI) Manuscript writing: All authors; (VII) Final approval of manuscript: All authors. \\ \#These authors contributed equally to this work. \\ Correspondence to: Li Yu; Xianghuai Xu. Department of Pulmonary and Critical Care Medicine, Tongji Hospital, Tongji University School of \\ Medicine, Shanghai 200065, China. Email: yuli0219@sina.com; 05849@tongji.edu.cn.
}

Background: This study aimed to investigate the reflux characteristics in patients with gastroesophageal reflux-related chronic cough (GERC) complicated by laryngopharyngeal reflux (LPR).

Methods: Patients with chronic cough were recruited. Reflux symptom index (RSI) scoring, cough symptom scoring, assessment of capsaicin cough sensitivity, and multichannel intraluminal impedance and pH monitoring (MII-pH) were performed.

Results: RSI score in GERC patients was significantly higher than that in patients with atopic cough (AC), cough variant asthma, eosinophilic bronchitis $(\mathrm{EB})$, and upper airway cough syndrome (UACS) $(\mathrm{P}<0.05)$. The RSI score in non-acid GERC patients was significantly higher than that in acid GERC patients $(\mathrm{P}=0.003)$. The cut-off value of the RSI score was defined as 19 during diagnosis of non-acid GERC. In the RSI $\geq 19$ group, there was more proximal reflux and more significant gas and non-acid reflux, and the efficacy of a combined use of baclofen or gabapentin was better than that of the RSI $<19$ group $(\mathrm{P}<0.05)$. The efficacy of proton pump inhibitor (PPI) at a routine dosage together with prokinetic agents in the RSI $<19$ group was better than that in the $\mathrm{RSI} \geq 19$ group $(\mathrm{P}=0.009)$.

Conclusions: LPR overlaps with GERC in part. GERC patients with higher RSI scores may present more proximal reflux, non-acid reflux, and gas reflux, and get better efficacy with neuromodulators (gabapentin or baclofen) used as an add-on therapy.

Keywords: Chronic cough; gastroesophageal reflux cough; multichannel intraluminal impedance and $\mathrm{pH}$ monitoring (MII-pH); reflux symptom index (RSI)

Submitted Jun 21, 2019. Accepted for publication Sep 24, 2019.

doi: 10.21037/atm.2019.09.162

View this article at: http://dx.doi.org/10.21037/atm.2019.09.162

\section{Introduction}

Gastroesophageal reflux disease refers to a condition where stomach contents come back up into the esophagus, resulting in either symptoms or complications. The characteristic esophageal symptoms of gastroesophageal reflux disease include regurgitation and heartburn. In some patients, there may be extra-esophageal symptoms such as cough, throat discomfort, and hoarseness (1).

Studies have demonstrated that gastroesophageal reflux-related chronic cough (GERC), a specific type of gastroesophageal reflux disease, is one of the most common causes of chronic cough (2-4). GERC accounts for $10-40 \%$ 
Table 1 Reflux symptom index scale

\begin{tabular}{lc}
\hline $\begin{array}{l}\text { Within the last month, how did the } \\
\text { following problems affect you? }\end{array}$ & $\begin{array}{r}\text { Score }(0=\text { no problem; } \\
5=\text { severe problem) }\end{array}$ \\
\hline Hoarseness or a problem with your voice. & $0 / 1 / 2 / 3 / 4 / 5$ \\
Clearing your throat. & $0 / 1 / 2 / 3 / 4 / 5$ \\
Excess throat mucus or postnasal drip & $0 / 1 / 2 / 3 / 4 / 5$ \\
Difficulty swallowing food, liquids, or pills & $0 / 1 / 2 / 3 / 4 / 5$ \\
Coughing after you ate or after lying & $0 / 1 / 2 / 3 / 4 / 5$ \\
down & \\
Breathing difficulties or choking episodes. & $0 / 1 / 2 / 3 / 4 / 5$ \\
Troublesome or annoying cough & $0 / 1 / 2 / 3 / 4 / 5$ \\
Sensation of something sticking in your & $0 / 1 / 2 / 3 / 4 / 5$ \\
throat or lump in your throat & \\
Heartburn, chest pain, indigestion, or & $0 / 1 / 2 / 3 / 4 / 5$ \\
stomachache, acid coming up & \\
Total & 45 \\
\hline
\end{tabular}

of chronic cough in Western countries and 6-20\% in China. With the development of diagnostic techniques, multichannel intraluminal impedance and $\mathrm{pH}$ monitoring (MII-pH) has been developed for the assessment of acid and non-acid gastroesophageal reflux episodes as well as their relationship with cough. Thus, MII-pH has been an effective tool in the diagnosis of GERC (5).

Recently, a study revealed that laryngopharyngeal reflux (LPR) was also a cause of chronic cough (6). Meanwhile, LPR was regarded as another special type of gastroesophageal reflux disease. Some symptoms of LPR (clearing throat, hoarseness, breathing difficulties, and globus hystericus) are considered extra-esophageal symptoms of gastroesophageal reflux disease. In 2002, Belafsky et al. designed a questionnaire of reflux symptom index (RSI). It was validated as a non-invasive, effective, and reliable tool for assessment of LPR. A Chinese version of RSI has also been developed and validated in Chinese patients $(7,8)$.

LPR and GERC, both causes of chronic cough, are special types of gastroesophageal reflux disease, but their symptoms are different. It is still unclear whether the site of reflux, nature of reflux contents, and treatment are different between LPR and GERC. This study aimed to investigate the reflux characteristics in patients with GERC complicated by LPR.

\section{Methods}

This was a prospective study. Patients with chronic cough were recruited from the Department of Pulmonary and Critical Care Medicine, Tongji Hospital of Tongji University between October 2016, and September 2017. Inclusion criteria were as follows: (I) patients were 16-80 years old; (II) the course of cough was longer than 8 weeks; (III) there were no other symptoms such as wheezing, hemoptysis, or fever; (IV) no rales were observed on lung auscultation; (V) chest X-ray or CT showed no abnormalities; (VI) lung function examination showed forced expiratory volume in one second (FEV1) $>80 \%$ of predicted and ratio of $\mathrm{FEV} 1 /$ forced vital capacity (FVC) $>70 \%$; (VII) patients agreed to receive RSI and a cough symptom scoring questionnaire. Exclusion criteria were as follows: (I) patients were pregnant or breast-feeding; (II) patients were smokers or had stopped smoking within the past two years; (III) patients had difficulties in reading or writing; (IV) patients' cough had markedly improved with specific treatment before the study; $(\mathrm{V})$ if patients were lost to follow-up or had incomplete medical information, the cause of cough was unclear, or patients refused to participate in the study, they were excluded from this study.

This study was approved by the Ethics Committee of Tongji Hospital of Tongji University [No. LL(H)-2016396] and registered as ChiCTR-DDD-17012587. Informed consent was obtained from each patient before the study.

\section{RSI score}

The RSI score was a self-assessment questionnaire (Table 1), which consisted of nine symptom-related questions concerning the severity of cough, factors triggering or deteriorating symptoms, concomitant post-nasal drip, regurgitation, and heartburn. The six-point scoring system (score 0 to 5) was employed, and the total score ranged from 0 to 45 . The higher the RSI score, the higher the probability of LPR; it is widely accepted that RSI $>13$ indicates a high probability of LPR (7).

\section{Cough symptom score}

The cough symptom score was obtained using the scale developed by Hsu et al. (9). This scale is divided into two parts (daytime and nighttime cough symptom scores), and 
the score ranges from 0 to 5 , with 0 being no cough and 5 being the most severe cough.

Capsaicin cough sensitivity was assessed with the modified Fujimura method according to the European Respiratory Society (ERS) guideline $(10,11)$. Patients received aerosol inhalation of capsaicin at an increasing concentration. The concentration of capsaicin when the number of coughs reached $\geq 2$ and $\geq 5$ was determined and used to reflect the cough sensitivity (C2 and C5, respectively).

\section{MII-pH}

MII-pH was performed as our own previous method $(12,13)$. Patients did not use anti-acid treatment during the examination or within two months before the examination. Abnormal gastroesophageal reflux was defined when the DeMeester score was $\geq 14.72$ and/or syndrome association probability (SAP) was $\geq 95 \%$ for abnormal acid gastroesophageal reflux or when the SAP was $\geq 95 \%$ for abnormal non-acid gastroesophageal reflux $(3,14)$.

Induced sputum was performed according to the protocol described previously (15). Patients inhaled a $4 \%$ hypertonic saline solution for 10-30 min through an ultrasonic nebulizer (YS9801, Yisheng Corp., Shanghai, China). The nose and mouth were cleaned before aerosol inhalation, and exertion cough was performed to expel the deep sputum which was then collected into a clean tank. The sputum without contamination by saliva was suspended in $0.1 \%$ DTT of 4 volumes, then incubated at $37{ }^{\circ} \mathrm{C}$ for $10 \mathrm{~min}$. The cell pellet was collected after centrifugation and smeared on glass slides. The air-dried preparations were stained with hematoxylin and eosin. The cells were differentiated by counting 400 nucleated cells according to the standard morphologic criteria.

\section{Detection of lung function and bronchial provocation test}

Pulmonary ventilation function examination and histamine bronchial provocation test were performed according to the guideline developed by the American Thoracic Society (ATS) (16). The bronchial provocation test is considered positive when $<7.8 \mu \mathrm{mol}$ histamine (cumulative dose) causes a 20\% fall in FEV1 (PD20-FEV1).

\section{Diagnostic criteria of GERC}

Patients who met the following criteria were diagnosed with GERC (14): (I) mainly daytime cough; (II) abnormal acid or non-acid reflux confirmed by MII-pH; (III) cough responsive to a stepwise anti-reflux therapy (17). A standard anti-reflux therapy (omeprazole $20 \mathrm{mg}$ twice daily plus domperidone $10 \mathrm{mg}$ thrice daily) was first introduced to suspected GERC patients. If no remission of cough was achieved, the dose of proton pump inhibitor (PPI) was doubled (omeprazole $80 \mathrm{mg}$ once daily) and continued for eight weeks. Patients who responded to the double-dosing were kept on this treatment until their cough resolved. If the cough was not resolved, then baclofen was introduced (omeprazole $20 \mathrm{mg}$ twice daily, baclofen 10-20 mg thrice daily) for four weeks, while domperidone was discontinued. After that, the remaining non-responders were escalated to omeprazole (20 $\mathrm{mg}$ twice daily) with gabapentin (100-300 mg thrice daily) for four weeks. If a favorable response occurred, the treatment was maintained until cough resolution was achieved.

\section{Study design}

After medical history review and physical examination, chest X-ray or CT scan, lung function examination, bronchial provocation test, an assay of cells in induced sputum and MII-pH were completed according to the diagnostic procedures of chronic cough, aiming to identify the causes of cough (14). All the diagnostic and treatment processes followed the 2015 Guideline for the Diagnosis and Treatment of Cough (14). Once upper airway cough syndrome (UACS), cough variant syndrome (CVA), eosinophilic bronchitis (EB), GERC or atopic cough (AC) was suspected, treatment targeting the cause of cough was initiated, aiming to confirm the diagnosis. In patients receiving $\mathrm{MII-pH}$, standard anti-reflux therapy was initiated when abnormal gastroesophageal reflux was present and continued for 2 months. If non-responsive, the dose of PPI was doubled, or neuromodulators (gabapentin or baclofen) as an add-on therapy were simultaneously administered $(12,13)$. GERC was diagnosed if the cough disappeared after combined therapy. When all the examination mentioned above showed negative, empirical therapy targeting the cause in the order of UACS, CVA, EB, AC, and GERC was performed. The diagnosis was confirmed or excluded according to the response to a specific therapy, and the potential cause was explored. During the therapy, patients were followed up by clinic visit once every 2 weeks. Patients with chronic cough received RSI scoring and post-therapy follow up. The primary endpoint was the findings of MII- 
Table 2 General clinical characteristics of patients with chronic cough

\begin{tabular}{lc}
\hline Variables & Outcome \\
\hline $\mathrm{N}(\mathrm{M} / \mathrm{F})$ & $139(51 / 88)$ \\
Age $(\mathrm{yr}, \bar{\chi} \pm \mathrm{S})$ & $45.0 \pm 20.0$ \\
Course of cough (month) & $12[55]$ \\
$\mathrm{RSI}$ score (point) & $13.1 \pm 6.4$ \\
Cough symptom score (point) & \\
Daytime & $3[1]$ \\
Nighttime & $2[1]$ \\
Lung function $(\%, \bar{\chi} \pm \mathrm{s})$ & \\
Predicted FEV1 $(\%)$ & $99.6 \pm 13.5$ \\
Predicted FVC $(\%)$ & $101.8 \pm 14.3$ \\
FEV1/FVC $\%$ & $82.9 \pm 7.4$ \\
\hline
\end{tabular}

$\mathrm{pH}$. The relationship between LPR and GERC was further assessed based on the characteristics of RSI and findings from MII-pH.

\section{Statistical analysis}

Data with normal distribution (such as age and lung function) were expressed as mean \pm standard deviation $(\overline{\mathrm{x}} \pm \mathrm{s})$. Data with abnormal distribution (such as course of cough, RSI, and cough symptom score) were presented as median (range interquartile). Data were compared with a $t$-test between the two groups once homogeneity test of variance was present or with Mann-Whitney $U$ test once heterogeneity of variance was present. Data were compared with Kruskal-Wallis test among groups once heterogeneity of variance was present. Statistical analysis was performed with SPSS version 20.0 (a relatively newer version). A value of $\mathrm{P}<0.05$ was considered statistically significant.

\section{Results}

\section{General characteristics}

During the study period, 163 patients met the inclusion criteria, 2 refused to participate in this study, 3 were lost to follow up, and 3 were excluded due to incomplete medical information. According to the results of examinations and the response to treatment, the cause of cough was unclear in 3 patients, double causes were found in 13 patients, and single cause was identified in 139 patients.

In 13 patients with two causes of cough, AC + GERC were noted in 8 patients, CVA + GERC in 2, EB + GERC in 2, and UACS + GERC in 1.

In 139 patients with single-cause chronic cough, GERC was found in 50 patients $(35.97 \%$; acid GERC in 28 patients and non-acid GERC in $17 ; 5$ patients refused to receive MII$\mathrm{pH}$ or had negative results of $\mathrm{MII}-\mathrm{pH}$, but were diagnosed with GERC according to the response to empirical antireflux therapy although acid or non-acid reflux could not be confirmed), CVA in 30 (21.58\%), EB in 20 (14.39\%), AC in $20(14.39 \%)$, ACEI-related cough in $3(2.16 \%)$, UACS in $12(8.63 \%)$, psychogenic cough in $2(1.44 \%)$, post-infection cough in $1(0.72 \%)$, and premature-beat-induced cough in $1(0.72 \%)$. The clinical characteristics of these patients are shown in Table 2 and Figure 1.

\section{RSI score in patients with chronic cough of different causes}

Among patients with different single-cause coughs, a significant difference in the RSI score was found $(\mathrm{H}=7.77$, $\mathrm{P}=0.000)$. The RSI score in GERC patients $\{17[18]\}$ was significantly higher than that in patients with AC [10.5 (9.5), $\mathrm{Z}=-3.529, \mathrm{P}=0.000]$, CVA [10 (3.25), $\mathrm{Z}=-4.853, \mathrm{P}=0.000]$, EB [10 (3.75), $Z=-3.059, P=0.002]$, UACS [11.75 (10.0), $\mathrm{Z}=-2.57, \mathrm{P}=0.01]$ and chronic cough of other causes [9.0 (4.0), $\mathrm{Z}=-2.983, \mathrm{P}=0.003]$. There was no significant difference among patients other than GERC patients (Figure 2).

\section{RSI score in patients with non-acid and acid GERC}

In the non-acid GERC group, the RSI score was significantly higher than that in the acid GERC group [21.00 (7.0) vs. 16.00 (6.5); $\mathrm{Z}=-2.956, \mathrm{P}=0.003]$. Also, in respect to the score of each question, the "cleaning throat" and "breathing difficulties" were more evident in the non-acid GERC patients than in the acid GERC patients (Table 3).

\section{Predictive value of RSI in non-acid GERC}

Since RSI was much higher in non-acid GERC, it was used to predict non-acid GERC. The area under the receiver operating characteristic curve of RSI was 0.765 , suggesting the predictive value of RSI. When the cut-off value was defined as 19, the Youden index was the highest (0.397), with a sensitivity of $64.71 \%$, a specificity of $75.00 \%$, a positive predictive value of $61.13 \%$, and a negative predictive value of $77.73 \%$ (Table 4 ). 


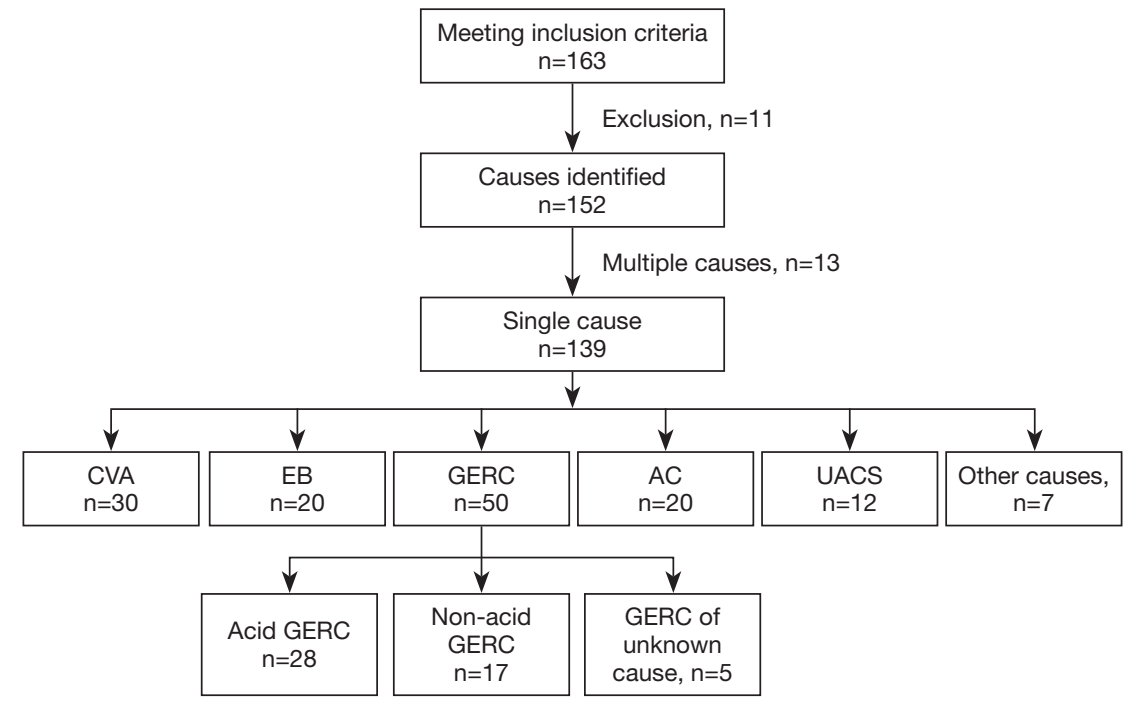

Figure 1 Causes of chronic cough. CVA, cough variant syndrome; EB, eosinophilic bronchitis; GERC, gastroesophageal reflux-related chronic cough; AC, atopic cough; UACS, upper airway cough syndrome.

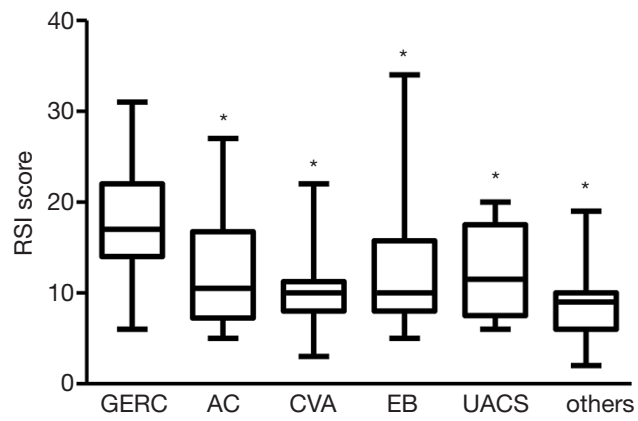

Figure 2 RSI score in patients with chronic cough of different causes. *, denotes $\mathrm{P}<0.05$ compared with GERC group, the distribution lines of the boxes from the bottom to the top mean first quartile, median, and third quartile. The very bottom of the vertical line on the boxes was the minimum. And the very top of the vertical line below the boxes was the maximum. RSI, reflux symptom index; GERC, gastroesophageal reflux-related chronic cough; AC, atopic cough; CVA, cough variant syndrome; EB, eosinophilic bronchitis; UACS, upper airway cough syndrome.

\section{Results of MII-pH in all GERC patients with different RSI scores}

All GERC patients who received MII-pH were divided into RSI $\geq 19$ group and RSI $<19$ groups. In the RSI $\geq 19$ group, there were 18 patients, of whom 7 had acid GERC (38.89\%), and 11 had non-acid GERC (61.11\%); in the RSI $<19$ group, acid GERC was found in 21 patients $(77.78 \%)$ and non-acid GERC in 6 patients (22.22\%). In the RSI $\geq 19$ group, the proportion of non-acid GERC patients was significantly higher than that of the RSI $<19$ group $\left(\chi^{2}=6.949\right.$, $\mathrm{P}=0.008$ ).

In addition, the DeMeester score in the RSI $\geq 19$ group was significantly lower than that in the RSI $<19$ group $(\mathrm{Z}=-2.944, \mathrm{P}=0.003)$; the incidences of non-acid SAP $(\mathrm{Z}=-2.771, \mathrm{P}=0.006)$, weakly acidic reflux $(\mathrm{Z}=-3.786$, $\mathrm{P}=0.000)$, gas reflux $(\mathrm{Z}=-2.179, \mathrm{P}=0.029)$, and proximal reflux event $(\mathrm{Z}=-2.073, \mathrm{P}=0.038)$ in the $\mathrm{RSI} \geq 19$ group were significantly higher than those in the RSI $<19$ group (Table 5).

\section{Therapeutic protocols in GERC patients with different RSI scores}

In the RSI $\geq 19$ group, the therapeutic efficacy of the combined use of baclofen or gabapentin was significantly better than that in the RSI $<19$ group, but the efficacy of a routine PPI dose plus prokinetic agents in the RSI $<19$ group was markedly better than that in the RSI $\geq 19$ group (Table 6).

\section{Discussion}

In the present study, the RSI score for GERC patients was significantly higher than in those with chronic cough caused by other causes, and this was more evident in patients with non-acid GERC. Also, in GERC patients with RSI $\geq 19$, 
Table 3 RSI score in patients with acid and non-acid GERC $\left\{\mathrm{M}\left[\mathrm{P}_{25}, \mathrm{P}_{75}\right]\right\}$

\begin{tabular}{|c|c|c|c|c|}
\hline Questions & Non-acid GERC $(n=17)$ & Acid GERC $(n=28)$ & Z & $\mathrm{P}$ \\
\hline Sensation of something sticking in your throat or lump in your throat & $3.00(1.00)$ & $2.50(2.00)$ & -1.406 & 0.160 \\
\hline Clearing your throat & $4.00(1.00)$ & $2.00(3.00)$ & -2.122 & 0.034 \\
\hline Excess throat mucus or postnasal drip & $2.00(2.00)$ & $1.50(3.00)$ & -1.296 & 0.195 \\
\hline Coughing after you ate or after lying down & $3.00(3.50)$ & $1.50(2.00)$ & -1.786 & 0.074 \\
\hline Distressing coughs $\mathrm{m}$ & $3.00(1.50)$ & $2.50(1.00)$ & -1.494 & 0.135 \\
\hline Difficulty swallowing food, liquids, or pills & $3.00(4.00)$ & $2.00(3.75)$ & -0.673 & 0.501 \\
\hline Heart burn, stomachache, chest pain & $0.00(2.00)$ & $1.00(3.00)$ & -1.197 & 0.231 \\
\hline
\end{tabular}

RSI, reflux symptom index; GERC, gastroesophageal reflux-related chronic cough.

Table 4 Predictive value of RSI in non-acid GERC at different cut-off values

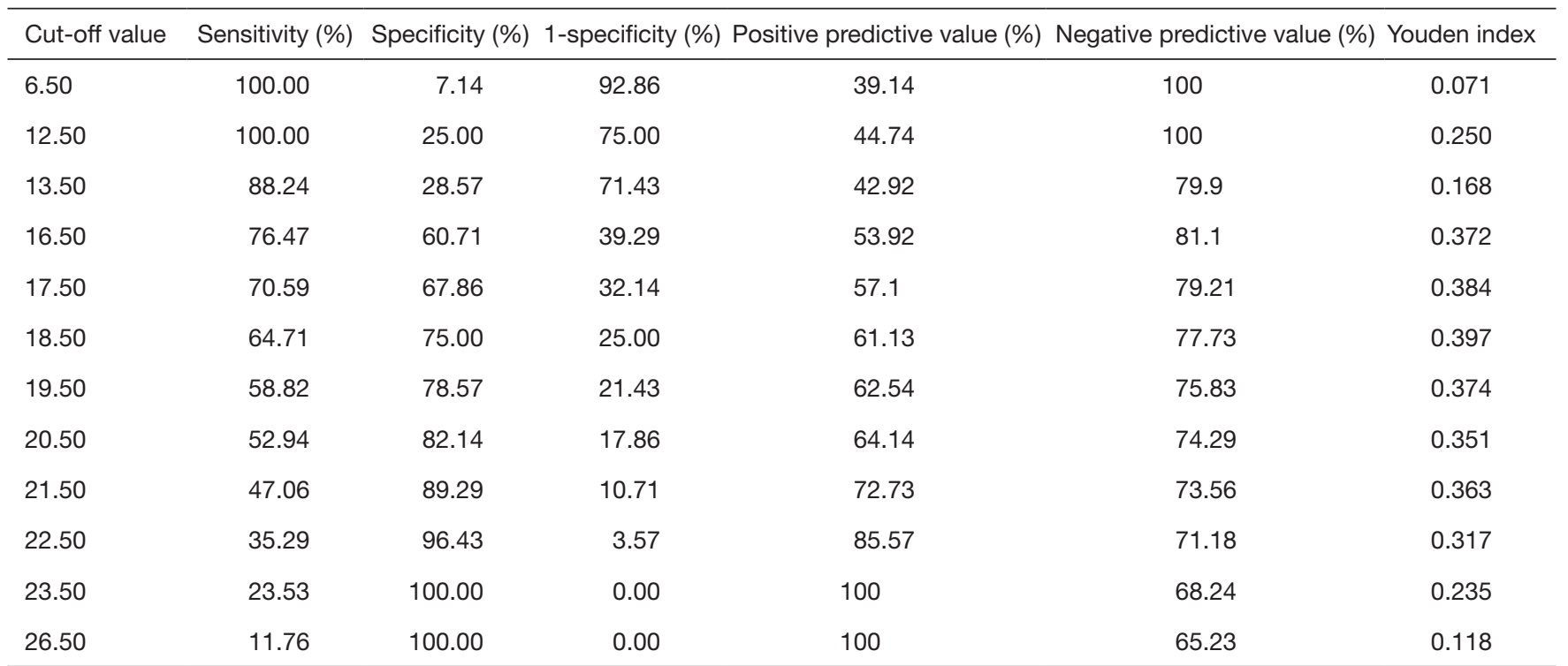

$\mathrm{RSI}$, reflux symptom index; GERC, gastroesophageal reflux-related chronic cough.

there was a higher proportion of proximal reflux, nonacid reflux, gas reflux, and better efficacy with concomitant baclofen or gabapentin.

LPR presents with a relatively high incidence. One largescale study showed $18 \%$ of patients presented symptoms of LPR, and $16 \%$ had symptoms of gastroesophageal reflux disease. This produces a significant economic burden to patients and society, and the annual cost for the treatment of extra-esophageal symptoms (including those of LPR) is as high as 50 billion (18). When reflux is present, the gastric contents may irritate the esophageal mucosa, pharynx, and larynx. However, the esophageal mucosa may protect against physiological reflux, which is not observed in the pharynx and larynx. Thus, gastroesophageal reflux can lead to LPR. Of note, there are no specific diagnostic criteria for LPR due to the limitations of diagnostic tools. The laryngopharyngeal manifestations of LPR and endoscopic findings are non-specific (19). Laryngopharyngeal 
monitoring and MII-pH are also unreliable. Pharyngeal reflux can be easily affected by swallowing. Pharyngeal symptoms are continuous, not acute and abrupt, which is different from cough and heartburn, and thus make recording SAP difficult. However, SAP is an important parameter in the diagnosis of LPR. Thus, MII-pH is insufficiently reliable for the diagnosis of LPR (20-23). In recent years, studies have revealed that the detection of pepsin in saliva has a high sensitivity and specificity in the diagnosis of LPR (24) and is closely related to the severity of laryngeal inflammation (25). However, other research has found no significant differences in the salivary

Table 5 Comparison of different variables in GERC with RSI $\geq 19$ and RSI $<19$

\begin{tabular}{lcc}
\hline Variables & $\begin{array}{c}\mathrm{RSI} \geq 19 \text { group } \\
(\mathrm{n}=18)\end{array}$ & $\begin{array}{c}\mathrm{RSI}<19 \text { group } \\
(\mathrm{n}=27)\end{array}$ \\
\hline DeMeester score & $5.8(14.5)$ & $42.6(111.9)^{*}$ \\
SAP for acid reflux (\%) & $51.8(81.4)$ & $67.4(88.9)$ \\
SAP for nonacid reflux (\%) & $78.7(95.0)$ & $0(68.0)^{*}$ \\
Acid reflux (n) & $25.4(29.0)$ & $28.6(27.0)$ \\
Weakly acidic reflux (n) & $38.5(31.0)$ & $13.7(25.0)^{*}$ \\
Weakly alkaline reflux (n) & $8.1(11.0)$ & $5.3(10.0)$ \\
Gas reflux (n) & $25.5(18.4)$ & $10.1(8.6)^{*}$ \\
Liquid reflux (n) & $14.9(21.5)$ & $15.3(22.1)$ \\
Mixed reflux (n) & $44.3(37.9)$ & $23.3(49.6)$ \\
Proximal extent (n) & $14.0(17.4)$ & $7.2(10.9)^{*}$ \\
Bolus exposure (\%) & $3.1(2.7)$ & $2.8(3.1)$ \\
Bolus clearance (s) & $8.1(10.7)$ & $11.3(6.9)$ \\
Acid exposure (\%) & $0.8(0.5)$ & $1.2(0.6)$ \\
Acid clearance (s) & $3.9(1.7)$ & $5.2(2.1)$ \\
\hline
\end{tabular}

*, $\mathrm{P}<0.05$ vs. RSI $\geq 19$. RSI, reflux symptom index; GERC, gastroesophageal reflux-related chronic cough. content of pepsin between LPR and normal controls, and no correlation of salivary pepsin with the number of reflux $(\mathrm{MII}-\mathrm{pH})$ and the reflux symptoms. Therefore, the value of detecting salivary pepsin is still controversial for the diagnosis of LPR (26-28). There is some evidence showing that RSI (based on important characteristic symptoms) and reflux finding score (based on laryngoscopic pathology plus questionnaire) can be used to diagnose LPR effectively (7). Belafsky et al. designed RSI, which consists of 9 questions (7). Available studies have shown that RSI is a non-invasive, simple, and effective tool that is easier to use in clinical practice as compared to reflux finding score (29). RSI is used to screen LPR based on clinical manifestations, and then anti-reflux therapy is employed, which has become an effective method for the confirmed diagnosis of LPR. RSI $>13$ may be used for the diagnosis of LPR, the effectiveness and reproducibility of which have been confirmed in some studies (30-32). Brauer et al. found that an RSI of 19 as a cut-off value had higher accuracy in the diagnosis of LPR in patients with concomitant allergic disease (33). In the present study, the 50 patients diagnosed with GERC had higher an RSI score than that of patients with other causes of chronic cough. In addition, $84 \%$ of the GERC patients had an RSI >13, with a good response to anti-reflux therapy. These findings suggest that LPR might also exist in these patients, indicating that there may be an overlap between LPR and GERC.

Furthermore, the study had more proximal reflux, higher incidence of non-acid reflux SAP, and more weakly acidic and gas reflux in GERC patients with an RSI $\geq 19$. Previous research revealed that proximal reflux might be present in patients with LPR and healthy controls. The reflux was mainly non-acid, which might be ascribed to the mucosal damage due to the LPR (33-36). It was found for the first time that GERC patients with concomitant LPR were more susceptible to proximal reflux and non-acid reflux, gas reflux was more common in these patients, and this type of reflux may be a critical cause of laryngopharyngeal injury

Table 6 Efficacy of different therapies in GERC with RSI $\geq 19$ and RSI $<19$

\begin{tabular}{lccc}
\hline Effective therapy & $\mathrm{RSI} \geq 19(\mathrm{n}=18)$ & $\mathrm{RSI}<19(\mathrm{n}=27)$ & $\chi^{2}$ value \\
\hline PPI at routine dose + prokinetic agents (standard) & $3(16.7 \%)$ & $15(55.6 \%)$ & 6.806 \\
PPI at double dose + prokinetic agents & $2(11.1 \%)$ & $8(29.6 \%)$ & 2.143 \\
Baclofen + PPI & $6(33.3 \%)$ & $2(7.4 \%)$ & 4.966 \\
Gabapentin + PPI & $7(38.9 \%)$ & $2(7.4 \%)$ & 6.143 \\
\hline
\end{tabular}

PPI, proton pump inhibitor; RSI, reflux symptom index; GERC, gastroesophageal reflux-related chronic cough. 
in GERC patients with LPR $\geq 19$. In this study, MII-pH was adopted as the golden standard in diagnosing GERC. Results showed that the RSI score was relatively accurate in predicting non-acid GERC. Non-acid GERC should be considered when the PSI score is $\geq 19$. Earlier studies have shown that patients with non-acid GERC mainly presented gas reflux (4). Together with the results of this study, we can speculate that proximal reflux is non-acid, which might be the main cause of LPR, indicating that there is an overlap between LPR and GERC, particularly non-acid GERC.

Pepsin is a main cause of laryngopharyngeal injury due to non-acid reflux $(34,35)$. It is widely accepted that pepsin is active only in an acidic environment. Johnston et al. found pepsin in a non-acid environment could also cause laryngopharyngeal damage. In a non-acid environment, the epithelial cells in the larynx and hypopharynx may undergo endocytose with pepsin in a receptor-mediated fashion; after entering the small vesicles with low $\mathrm{pH}$ value, pepsin becomes active and exerts effects. When the $\mathrm{pH}$ value is 7 , pepsin may cause damage to cells, which is related to the laryngoscopic findings and symptoms, suggesting that the intracellular environment may activate pepsin. Johnston et al. also confirmed that the $\mathrm{pH}$ value in the Golgi body was 5 , and $40 \%$ of pepsin in the Golgi body could be activated. Therefore, inactivated pepsin can be re-activated in the intracellular compartments (such as the Golgi body) with low $\mathrm{pH}$ value after endocytosis, causing cell injury (36). In addition, in vitro studies also indicate that incubation of pepsin with human hypopharyngeal cells in a non-acid environment may induce the production of some pro-inflammatory cytokines such as CCL20, CCL26, IL8, and these cytokines are also related to reflux-induced esophagitis. These findings imply that pepsin is a critical cause of laryngopharyngeal inflammation, even in a nonacid environment (37). This also explains why, in the present study, most LPR patients that had non-acid reflux also suffered a series of laryngopharyngeal injury symptoms.

Although anti-reflux therapy was performed in all patients with GERC, the PPI at double dose achieved a better success rate in GERC patients with an RSI $<19$, but more GERC patients with an RSI $\geq 19$ achieved better cough control after concomitant use of gabapentin or baclofen. The treatments for acid reflux and non-acid reflux are different. PPI may achieve better efficacy for acid reflux, while additional prokinetic agents or even esophageal sphincter relaxants (such as gabapentin and baclofen) is needed for non-acid reflux. In GERC patients with an RSI $<19$, MII-pH had a higher DeMeester score, which was characterized by acid reflux, and thus PPI at a higher dose achieved better efficacy. However, in GERC patients with an RSI $\geq 19$, weakly acidic reflux was mainly present, and therefore additional gabapentin or baclofen achieved better efficacy, which was consistent with findings from our previous studies. Our findings suggest that proximal reflux and non-acid reflux may be predicted if GERC patients have an RSI $\geq 19$, and concomitant use of neuromodulators, including gabapentin and baclofen, is needed for the treatment $(38,39)$.

There are some limitations to this study. First, concomitant use of laryngopharyngeal MII-pH may be better to directly and accurately confirm the diagnosis of LPR in GERC patients. Second, we were unable to directly evaluate the inhibitory efficacy of gabapentin and baclofen on acid or non-acid reflux as the patients refused to undergo a repeat invasive MII-pH study at the end of the treatment period. Moreover, it is difficult to exclude the contribution of the nonspecific non-reflux-related antitussive activity of neuromodulators. Nevertheless, our previous study showed that gabapentin and baclofen have similar therapeutic efficacy for suspected refractory GERC; the obvious parallel reduction in GerdQ suggests that the improvement in cough, at least in part, can be attributed to the blockade of abnormal reflux $(38,39)$.

In summary, this study revealed that GERC patients with a high RSI score are more likely to have proximal reflux (reflux more close to the throat) indicating an overlap between LPR and GERC, and these GERC patients are always characterized by non-acid reflux and gas reflux. Neuromodulators (gabapentin or baclofen) can be used as an add-on therapy to GERC patients with a higher RSI score.

\section{Acknowledgments}

Funding: This study was supported by the National Natural Science Foundation of China (No. 81770097 and 81670092), the Project of Science and Technology Commission of Shanghai Municipality (No. 17411970800), and the Shanghai Sailing Program (No. 19YF1444100).

\section{Footnote}

Conflicts of Interest: The authors have no conflicts of interest to declare.

Ethical Statement: The authors are accountable for all 
aspects of the work in ensuring that questions related to the accuracy or integrity of any part of the work are appropriately investigated and resolved. This study was approved by the Ethics Committee of Tongji Hospital of Tongji University [No. LL(H)-2016-396] and registered as ChiCTR-DDD-17012587. Informed consent was obtained from each patient before the study.

\section{References}

1. Vakil N, van Zanten SV, Kahrilas P, et al. The Montreal definition and classification of gastroesophageal reflux disease: a global evidence-based consensus. Am J Gastroenterol 2006;101:1900-20; quiz 1943.

2. Kahrilas PJ, Altman KW, Chang AB, et al. Chronic Cough Due to Gastroesophageal Reflux in Adults: CHEST Guideline and Expert Panel Report. Chest 2016;150:1341-60.

3. Lai K, Chen R, Lin J, et al. A prospective, multicenter survey on causes of chronic cough in China. Chest 2013;143:613-20.

4. Yu L, Wei WL, Lu HJ, et al. Changes in the spectrum and frequency of causes for chronic cough: a retrospective analysis. Zhonghua Jie $\mathrm{He} \mathrm{He} \mathrm{Hu} \mathrm{Xi} \mathrm{Za}$ Zhi 2009;32:414-7.

5. Xu X, Chen Q, Liang S, et al. Comparison of gastroesophageal reflux disease questionnaire and multichannel intraluminal impedance $\mathrm{pH}$ monitoring in identifying patients with chronic cough responsive to antireflux therapy. Chest 2014;145:1264-70.

6. Morice AH. Is reflux cough due to gastroesophageal reflux disease or laryngopharyngeal reflux? Lung 2008;186 Suppl 1:S103-6.

7. Belafsky PC, Postma GN, Koufman JA. Validity and reliability of the reflux symptom index (RSI). J Voice 2002;16:274-7.

8. Zheng JY, Zhang LH, Li JJ, et al. Chinese version of the reflux symptom index was evaluated for reliability and validity. Zhonghua Er Bi Yan Hou Tou Jing Wai Ke Za Zhi 2012;47:894-8.

9. Hsu JY, Stone RA, Logan-Sinclair RB, et al. Coughing frequency in patients with persistent cough: assessment using a 24 hour ambulatory recorder. Eur Respir J 1994;7:1246-53.

10. Fujimura M, Kasahara K, Kamio Y, et al. Female gender as a determinant of cough threshold to inhaled capsaicin. Eur Respir J 1996;9:1624-6.

11. Morice AH, Fontana GA, Belvisi MG, et al. ERS guidelines on the assessment of cough. Eur Respir J 2007;29:1256-76.

12. Qiu Z, Yu L, Xu S, et al. Cough reflex sensitivity and airway inflammation in patients with chronic cough due to non-acid gastro-oesophageal reflux. Respirology 2011;16:645-52.

13. Xu XH, Yang ZM, Chen Q, et al. Therapeutic efficacy of baclofen in refractory gastroesophageal reflux-induced chronic cough. World J Gastroenterol 2013;19:4386-92.

14. Asthma Group Branch of Respiratory Disease Chinese Medical Association. Guideline for the Diagnosis and Treatment of Cough (2015). Chin J Tubercul Resp Dis 2016:323-54.

15. Yu L, Wei W, Wang L, et al. Upper-airway cough syndrome with latent eosinophilic bronchitis. Lung 2010;188:71-6.

16. Asthma Group Branch of Respiratory Disease Chinese Medical Association. Guideline for the Prevention and Treatment of Bronchial Asthma. Chin J Tubercul Resp Dis 2016:675-97.

17. Dong R, Xu X, Yu L, et al. Randomised clinical trial: gabapentin vs baclofen in the treatment of suspected refractory gastro-oesophageal reflux-induced chronic cough. Aliment Pharmacol Ther 2019;49:714-22.

18. Sidhwa F, Moore A, Alligood E, et al. Diagnosis and Treatment of the Extraesophageal Manifestations of Gastroesophageal Reflux Disease. Ann Surg 2017;265:63-7.

19. Hicks DM, Ours TM, Abelson TI, et al. The prevalence of hypopharynx findings associated with gastroesophageal reflux in normal volunteers. J Voice 2002;16:564-79.

20. Kim SI, Kwon OE, Na SY, et al. Association between 24hour combined multichannel intraluminal impedance$\mathrm{pH}$ monitoring and symptoms or quality of life in patients with laryngopharyngeal reflux. Clin Otolaryngol 2017;42:584-91.

21. Lee YC, Kwon OE, Park JM, et al. Do laryngoscopic findings reflect the characteristics of reflux in patients with laryngopharyngeal reflux? Clin Otolaryngol 2018;43:137-43.

22. Pavic I, Babic I, Cepin Bogovic J, et al. The importance of combined 24-hour multichannel intraluminal impedance-pH monitoring in the evaluation of children with suspected laryngopharyngeal reflux. Clin Otolaryngol 2017;42:544-9.

23. Zerbib F, Dulery C. Facts and Fantasies on Extraesophageal Reflux: A Gastroenterologist Perspective. J Clin Gastroenterol 2017;51:769-76.

24. Saritas Yuksel E, Hong SK, Strugala V, et al. Rapid salivary 
pepsin test: blinded assessment of test performance in gastroesophageal reflux disease. Laryngoscope 2012;122:1312-6.

25. Spyridoulias A, Lillie S, Vyas A, et al. Detecting laryngopharyngeal reflux in patients with upper airways symptoms: Symptoms, signs or salivary pepsin? Respir Med 2015;109:963-9.

26. Dy F, Amirault J, Mitchell PD, et al. Salivary Pepsin Lacks Sensitivity as a Diagnostic Tool to Evaluate Extraesophageal Reflux Disease. J Pediatr 2016;177:53-8.

27. Wang J, Zhao Y, Ren J, et al. Pepsin in saliva as a diagnostic biomarker in laryngopharyngeal reflux: a metaanalysis. Eur Arch Otorhinolaryngol 2018;275:671-8.

28. Yadlapati R, Adkins C, Jaiyeola DM, et al. Abilities of Oropharyngeal pH Tests and Salivary Pepsin Analysis to Discriminate Between Asymptomatic Volunteers and Subjects With Symptoms of Laryngeal Irritation. Clin Gastroenterol Hepatol 2016;14:535-42.e2.

29. Watson NA, Kwame I, Oakeshott P, et al. Comparing the diagnosis of laryngopharyngeal reflux between the reflux symptom index, clinical consultation and reflux finding score in a group of patients presenting to an ENT clinic with an interest in voice disorders: a pilot study in thirtyfive patients. Clin Otolaryngol 2013;38:329-33.

30. Printza A, Kyrgidis A, Oikonomidou E, et al. Assessing laryngopharyngeal reflux symptoms with the Reflux Symptom Index: validation and prevalence in the Greek population. Otolaryngol Head Neck Surg 2011;145:974-80.

31. Salihefendic N, Zildzic M, Cabric E. Laryngopharyngeal

Cite this article as: Yu Y, Wen S, Wang S, Shi C, Ding H, Qiu Z, Xu X, Yu L. Reflux characteristics in patients with gastroesophageal reflux-related chronic cough complicated by laryngopharyngeal reflux. Ann Transl Med 2019;7(20):529. doi: 10.21037/atm.2019.09.162
Reflux Disease - LPRD. Med Arch 2017;71:215-8.

32. Yang J, Dehom S, Sanders S, et al. Treating laryngopharyngeal reflux: Evaluation of an antireflux program with comparison to medications. Am J Otolaryngol 2018;39:50-5.

33. Brauer DL, Tse KY, Lin JC, et al. The Utility of the Reflux Symptom Index for Diagnosis of Laryngopharyngeal Reflux in an Allergy Patient Population. J Allergy Clin Immunol Pract 2018;6:132-8.e1.

34. Johnston N, Knight J, Dettmar PW, et al. Pepsin and carbonic anhydrase isoenzyme III as diagnostic markers for laryngopharyngeal reflux disease. Laryngoscope 2004;114:2129-34.

35. Johnston N, Yan JC, Hoekzema CR, et al. Pepsin promotes proliferation of laryngeal and pharyngeal epithelial cells. Laryngoscope 2012;122:1317-25.

36. Johnston N, Dettmar PW, Bishwokarma B, et al. Activity/ stability of human pepsin: implications for reflux attributed laryngeal disease. Laryngoscope 2007;117:1036-9.

37. Samuels TL, Johnston N. Pepsin as a causal agent of inflammation during nonacidic reflux. Otolaryngol Head Neck Surg 2009;141:559-63.

38. Xu X, Lv H, Yu L, et al. A stepwise protocol for the treatment of refractory gastroesophageal reflux-induced chronic cough. J Thorac Dis 2016;8:178-85.

39. Xu X, Yu L, Chen Q, et al. Diagnosis and treatment of patients with nonacid gastroesophageal reflux-induced chronic cough. J Res Med Sci 2015;20:885-92. 